\title{
CONVERGENCE OF THE TWO-POINT UPSTREAM WEIGHTING SCHEME
}

\author{
MARIE-CLAUDE VIALLON
}

\begin{abstract}
Convergence to the entropy solution of a second-order scheme for the resolution of scalar hyperbolic conservation laws is studied. We consider the case of the so-called two-point upstream weighting scheme, widely used in petroleum engineering, to solve fluid flows in porous media problems. We prove convergence of the two-point upstream weighting scheme to the entropy solution for both discrete and semidiscrete approximations.
\end{abstract}

\section{INTRODUCTION}

Recently, much research has been done to solve hyperbolic conservation laws by the use of high-order numerical schemes. However, the two-point upstream weighting scheme $[8,9,13,1,2]$, which is a second-order finite difference scheme, was used in petroleum engineering for some twenty years without, to our knowledge, any mathematically rigorous theory (except indications of linear stability [2]). Several variants have already been numerically tested for fluid flows in porous media problems (saturation or concentration equation) and give excellent results. The goal of this paper is to prove convergence of the two-point upstream weighting scheme to the entropy solution of the fully discrete and semidiscrete (that is to say, continuous in time) versions.

It is worth noticing that, in the fully discrete case, the convergence is obtained under sharp CFL conditions; and in the semidiscrete case, the convergence is proved thanks to the fact that the numerical flux of the scheme is globally Lipschitz under monotonicity and regularity assumptions.

\section{Preliminaries}

We consider the scalar hyperbolic conservation law

$$
u_{t}+(f(u))_{x}=0 \text { with } x \in \mathbb{R} \text {, and } t \in[0, T), T>0 \text {, }
$$

with initial condition $u(x, 0)=u_{0}(x)$.

We assume $f \in C^{2}$ and smooth initial data. It is well known, however, that solutions of (1.1) may be discontinuous, and we have to define a weak solution

Received January 15, 1990; revised September 26, 1990.

1980 Mathematics Subject Classification (1985 Revision). Primary 65M10, 35A40. 
of (1.1):

$$
\left\{\begin{array}{l}
u \in L_{\mathrm{loc}}^{\infty}(\mathbb{R} \times[0, T)), \\
\forall \varphi \in C_{0}^{1}(\mathbb{R} \times[0, T)), \\
\quad \int_{0}^{T} \int_{\mathbb{R}}\left(u \varphi_{t}+f(u) \varphi_{x}\right)+\int_{\mathbb{R}} u_{0}(x) \varphi(x, 0) d x=0 .
\end{array}\right.
$$

Solutions of (1.2) are not necessarily unique, and we are interested in the physical one, the so-called entropy solution, that is characterized by the Lax entropy condition

$$
(U(u))_{t}+(F(u))_{x} \leq 0 \text { in the weak sense },
$$

for all entropy pairs $(U, F)$ associated with (1.1), where $U$ is convex.

For scalar convex conservation laws whose solution is of bounded variation, R. J. Di Perna [3] proves it is sufficient that the Lax entropy condition be satisfied for a single strictly convex entropy function $U$.

We now define a fully discretized conservative and consistent finite difference scheme: Let $\Delta x$ be the space step, $\Delta t$ be the time step, and $\lambda$ be the ratio $\Delta t / \Delta x$. We decompose the interval $[0, T)$ into $L$ (integer) subintervals $J^{n}=$ $\left[t^{n}, t^{n+1}\right.$ ), with $n \in \mathbb{N}$ and $n=0, \ldots, L-1$, where $t^{n}=n \Delta t$ (we assume $T=L \Delta t)$. We decompose the real axis into intervals $I_{i}=\left(x_{i-1 / 2}, x_{i+1 / 2}\right)$, where $x_{i+1 / 2}=x_{i}+\Delta x / 2$ and $x_{i}=i \Delta x$, for $i \in \mathbb{Z}$. We denote by $u_{i}^{n}$ an approximation of the average value of the solution $u$ in $K_{i}^{n}=I_{i} \times J^{n}$. A conservative and consistent fully discretized and explicit finite difference scheme is

$$
u_{i}^{n+1}=u_{i}^{n}-\lambda\left(g_{i+1 / 2}^{n}-g_{i-1 / 2}^{n}\right) \text {, }
$$

where $g_{i+1 / 2}^{n}=g\left(u_{i-k+1}^{n}, \ldots, u_{i+k}^{n}\right), g$ being the numerical flux of the scheme, assumed to be continuous, such that $g(u, \ldots, u)=f(u)$.

We assume $u_{0} \in L_{\text {loc }}^{1}(\mathbb{R})$ and we choose $u_{i}^{0}=\frac{1}{\Delta x}\left(\int_{I_{i}} u_{0}(x) d x\right), i \in \mathbb{Z}$. The approximate solution of $(1.1)$, denoted by $u_{\Delta}(x, t)$, is then a piecewise constant function defined by $u_{\Delta}(x, t)=u_{i}^{n}$ if $(x, t) \in K_{i}^{n}$.

Next we define a semidiscretized conservative and consistent finite difference scheme: We discretize the real axis as above, and for all $t \in[0, T)$, we denote by $u_{i}(t)$ an approximation of the average value of the solution in the interval $I_{i}$. A conservative and consistent semidiscrete scheme is a system of differential equations

$$
\frac{d u_{i}}{d t}+\frac{1}{\Delta x}\left(g_{i+1 / 2}-g_{i-1 / 2}\right)=0
$$

where $g_{i+1 / 2}=g\left(u_{i-k+1}, \ldots, u_{i+k}\right), g$ being continuous, such that $g(u, \ldots, u)=f(u)$.

We assume $u_{0} \in L_{\text {loc }}^{1}(\mathbb{R})$ and we define $u_{i}(0)=\frac{1}{\Delta x}\left(\int_{I_{i}} u_{0}(x) d x\right), i \in \mathbb{Z}$. The approximate solution is the step function $u_{\Delta}(x, t)=u_{i}(t)$ when $x \in I_{i}$, with $t \in[0, T)$. 
The two-point upstream weighting scheme has the following property: it is a conservative and consistent finite difference scheme, fully determined by its numerical flux denoted by $f^{\mathrm{DA}}$. We shall prove that the sequence $\left(u_{\Delta}\right)$ computed by the scheme converges to a weak solution of (1.1) as the discretization step $\Delta x$ tends to zero. Then we prove that this weak solution is the unique entropy solution by establishing an entropy inequality.

We consider separately the fully discrete case and the semidiscrete case.

\section{Definition of THE NUMerical FLUX $f^{\text {DA }}$}

In order to approach the value of the flux at the point $x_{i+1 / 2}$, the scheme calculates an approximation of $u\left(x_{i+1 / 2}\right)$ by using a linear interpolation constructed on $u_{i-1}$ and $u_{i}$ if the flow comes from $i$ to $i+1$, and on $u_{i+2}$ and $u_{i+1}$ otherwise.

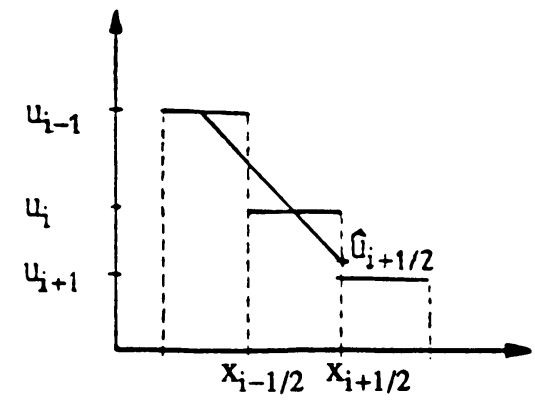

FIGURE 1

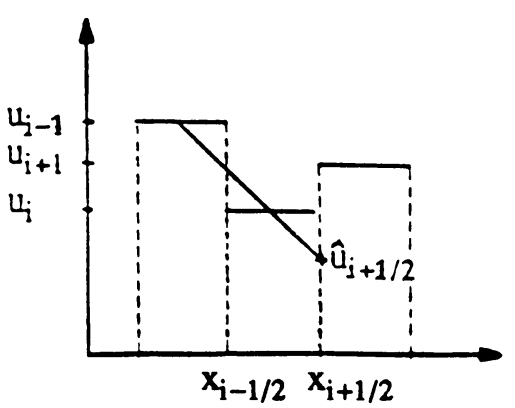

FIGURE 2

In petroleum engineering, where this scheme is widely used, the flow direction is easily determined by physical considerations. That is why, out of this context, to define the flow direction, we will proceed in a more general way, by using the Godunov flux $f^{\mathrm{G}}$, denoting $f_{i+1 / 2}^{\mathrm{G}}=f^{\mathrm{G}}\left(u_{i+1}, u_{i}\right)$ :

$$
\left\{\begin{array}{l}
\text { The flow is }\left\{\begin{array}{l}
\text { coming from } i \text { to } i+1, \text { if } f_{i+1 / 2}^{\mathrm{G}}=f\left(u_{i}\right), \\
\text { coming from } i+1 \text { to } i, \text { if } f_{i+1 / 2}^{\mathrm{G}}=f\left(u_{i+1}\right),
\end{array}\right. \\
\text { else the direction of the flow is undetermined. }
\end{array}\right.
$$

Then we define the linear interpolate $\hat{u}_{i+1 / 2}$ and $u_{i+1 / 2}$ by

$$
\begin{cases}\hat{u}_{i+1 / 2}=\frac{3}{2} u_{i}-\frac{1}{2} u_{i-1} & \text { if } f_{i+1 / 12}^{\mathrm{G}}=f\left(u_{i}\right), \\ u_{i+1 / 2}=\frac{3}{2} u_{i+1}-\frac{1}{2} u_{i+2} & \text { if } f_{i+1 / 2}^{\mathrm{G}}=f\left(u_{i+1}\right),\end{cases}
$$

if the direction of the flow is well determined. A natural solution when the direction of the flow is undetermined is to use the Godunov scheme and to define $f_{i+1 / 2}^{\mathrm{DA}}=f_{i+1 / 2}^{\mathrm{G}}$.

Finally, a slope limiter has to be introduced in order to keep the monotonicity of the solution. That is to say, we must enforce the interpolates to be located 
between $u_{i}$ and $u_{i+1}$. We define the corrected interpolate $\bar{u}_{i+1 / 2}$, associated with a flow coming from the left, in the following way:

$$
\bar{u}_{i+1 / 2}= \begin{cases}\min \left(\hat{u}_{i+1 / 2}, u_{i+1}\right) & \text { if } u_{i-1} \leq u_{i} \leq u_{i+1}, \\ \max \left(\hat{u}_{i+1 / 2}, u_{i+1}\right) & \text { if } u_{i+1} \leq u_{i} \leq u_{i-1} \\ u_{i} & \text { else. }\end{cases}
$$

(We define the corrected interpolate $\underline{u}_{i+1 / 2}$, when the flow comes from the right, in the same way.)

The flux $f_{i+1 / 2}^{\mathrm{DA}}$ could be chosen equal to $f\left(\bar{u}_{i+1 / 2}\right)$ or $f\left(\underline{u}_{i+1 / 2}\right)$ according to the direction flow. But it is better to consider $\bar{u}_{i+1 / 2}\left(u_{i}\right)$ as the left value at $x_{i+1 / 2}$, and $u_{i+1}\left(\underline{u}_{i+1 / 2}\right)$ as the right value at $x_{i+1 / 2}$, to solve the associated Riemann's problem, if the flow comes from the left (right). This gives rise to the definition of $f_{i+1 / 2}^{\mathrm{DA}}$ :

$$
f_{i+1 / 2}^{\mathrm{DA}}=\left\{\begin{array}{l}
f^{\mathrm{G}}\left(u_{i+1}, \bar{u}_{i+1 / 2}\right) \text { if } f^{\mathrm{G}}\left(u_{i+1}, u_{i}\right)=f\left(u_{i}\right), \\
f^{\mathrm{G}}\left(\underline{u}_{i+1 / 2}, u_{i}\right) \text { if } f^{\mathrm{G}}\left(u_{i+1}, u_{i}\right)=f\left(u_{i+1}\right), \\
f\left(\xi_{i+1 / 2}\right) \text { if } f^{\mathrm{G}}\left(u_{i+1}, u_{i}\right)=f\left(\xi_{i+1 / 2}\right) \notin\left\{f\left(u_{i}\right), f\left(u_{i+1}\right)\right\}
\end{array}\right.
$$

The two-point upstream weighting scheme is a five-point scheme in one dimension.

The scheme is second-order accurate in space, away from the discontinuities (see $[1,2,9])$, in smooth parts of the solution. For instance, if $f$ is increasing, writing $f\left(\hat{u}_{i+1 / 2}\right)=f\left(u_{i}+\frac{1}{2}\left(u_{i}-u_{i-1}\right)\right)$ and using Taylor series expansion, allows one to prove that

$$
\begin{aligned}
\frac{1}{\Delta x}\left(f_{i+1 / 2}^{\mathrm{DA} . n}-f_{i-1 / 2}^{\mathrm{DA}, n}\right) & =\frac{1}{\Delta x}\left(f\left(\hat{u}_{i+1 / 2}^{n}\right)-f\left(\hat{u}_{i-1 / 2}^{n}\right)\right) \\
& =\frac{\partial}{\partial x}\left(f\left(u\left(x_{i}, t^{n}\right)\right)\right)+\Delta x \varepsilon(\Delta x) .
\end{aligned}
$$

But it is worth noticing that the scheme is only first-order accurate in time.

As far as the convergence of the scheme is concerned, the only theoretical result we have is a stability study when $f(u)=u$ [2], using von Neumann's method. It is proved in [2] that the two-point upstream weighting scheme can be considered as "stable" under the condition $\lambda \leq 2 / 3$.

\section{Part I. Convergence of the Fully Discrete \\ Two-Point Upstream Weighting Scheme to THE ENTROPy Solution}

\section{Convergence to a Weak SOlution}

A standard way to prove the convergence of the scheme (1.4) to a weak solution is (assuming $u_{0} \in L^{1}(\mathbb{R}) \cap B V(\mathbb{R})$ ) to verify that the total variation of the computed solution is decreasing (TVD), that is to say, for $n=0, \ldots, L-1$ :

$$
V T\left(u_{\Delta}\left(\cdot, t^{n+1}\right)\right)=\sum_{i \in \mathbb{Z}}\left|u_{i+1}^{n+1}-u_{i}^{n+1}\right| \leq V T\left(u_{\Delta}\left(\cdot, t^{n}\right)\right),
$$


that the scheme is $L^{\infty}$-stable, that is to say, there exists a constant $C\left(u_{0}\right)>0$ which depends only on the initial condition $u_{0}$ such that

$$
\left\|u_{\Delta}(\cdot, t)\right\|_{L^{\infty}(\mathbb{R})}=\sup _{i \in \mathbb{Z} ; n=0, \ldots, L-1}\left|u_{i}^{n}\right| \leq C\left(u_{0}\right) \text { for all } t \in[0, T),
$$

and the numerical flux is "locally Lipschitz" " (see [11]), that is to say, there exists a constant $C$, depending only on the scheme's $L^{\infty}$-stability boundedness, such that

$$
\left|g_{i+1 / 2}-g_{i-1 / 2}\right| \leq C\left\|\left(u_{i-k+1}, \ldots, u_{i+k}\right)-\left(u_{i-k}, \ldots, u_{i+k-1}\right)\right\|_{\mathbb{R}^{2 k}} .
$$

We use A. Harten's approach [4] to prove that a finite difference scheme (1.4) is TVD. We have to write the scheme in incremental form, that is to say, finding functions $C$ and $D$ such that

$$
\lambda\left(g_{i+1 / 2}^{n}-g_{i-1 / 2}^{n}\right)=-C_{i+1 / 2}\left(u_{i+1}^{n}-u_{i}^{n}\right)+D_{i-1 / 2}\left(u_{i}^{n}-u_{i-1}^{n}\right)
$$

with $C_{i+1 / 2}=C\left(u_{i-k+1}^{n}, \ldots, u_{i+k}^{n}\right)$ and $D_{i+1 / 2}=D\left(u_{i-k+1}^{n}, \ldots, u_{i+k}^{n}\right)$, hence, with (3.4), the scheme (1.4) becomes

$$
u_{i}^{n+1}=u_{i}^{n}+C_{i+1 / 2}\left(u_{i+1}^{n}-u_{i}^{n}\right)-D_{i-1 / 2}\left(u_{i}^{n}-u_{i-1}^{n}\right) .
$$

According to [4], the scheme (3.5) is TVD if $C_{i+1 / 2} \geq 0, D_{i+1 / 2} \geq 0$, and $C_{i+1 / 2}+D_{i+1 / 2} \leq 1$, for $i \in \mathbb{Z}$. It follows also from (3.5) that, if $C_{i+1 / 2} \geq 0$, $D_{i+1 / 2} \geq 0$, and $C_{i+1 / 2}+D_{i-1 / 2} \leq 1$, for all $i \in \mathbb{Z}$, then we have the maximum principle

$$
\forall i \in \mathbb{Z}, \forall n=0, \ldots, L-1, \quad \inf _{i \in \mathbb{Z}} u_{i}^{n} \leq u_{i}^{n+1} \leq \sup _{i \in \mathbb{Z}} u_{i}^{n},
$$

which proves that the scheme is $L^{\infty}$-stable if we assume $u_{0} \in L^{\infty}(\mathbb{R})$.

Hence, the proofs of the convergence theorems stated below will be totally based on the determination of the incremental coefficients $C_{i+1 / 2}$ and $D_{i+1 / 2}$.

Theorem 3.1. If $u_{0} \in L^{1}(\mathbb{R}) \cap L^{\infty}(\mathbb{R}) \cap B V(\mathbb{R})$, then for a convex function $f$, under the CFL condition $\lambda \sup \left\{\left|f^{\prime}\left(u_{i}\right)\right|, i \in \mathbb{Z}\right\} \leq \frac{1}{3}$, the fully discrete two-point upstream weighting scheme converges to a weak solution of the problem (1.1).

Theorem 3.2. If $u_{0} \in L^{1}(\mathbb{R}) \cap L^{\infty}(\mathbb{R}) \cap B V(\mathbb{R})$, then for a monotone function $f$, under the CFL condition $\lambda \sup \left|f^{\prime}\right| \leq \frac{2}{3}$, the fully discrete two-point upstream weighting scheme converges to a weak solution of the problem (1.1).

Remark 1. We remark that we obtain sharp CFL conditions for convergence, probably because the scheme is second-order accurate in space (except near critical points). It is worth noticing that the assumption of monotonicity of $f$ gives a CFL condition two times the CFL condition associated with a convex function; the result of Theorem 3.2 is consistent with the von Neumann stability condition given in [2]. 
Remark 2. The case $f$ monotone is the usual case in petroleum engineering simulations.

\section{Proof of Theorems 3.1 and 3.2}

Stating the incremental decomposition of the two-point upstream weighting scheme, we seek the coefficients $C_{i+1 / 2}$ and $D_{i+1 / 2}$. We prove they are positive, they satisfy $C_{i+1 / 2}+D_{i \pm 1 / 2} \leq 1$ under the CFL condition, and they depend only on the scheme's $L^{\infty}$-stability boundedness. To bound $C_{i+1 / 2}$ and $D_{i+1 / 2}$, we have to first establish a lemma. (Because of complexity of notation, we will in the following omit the exponent $n$.)

\subsection{Preliminary computation.}

Lemma 4.1. The four ratios

$$
\frac{\bar{u}_{i+1 / 2}-\bar{u}_{i-1 / 2}}{u_{i}-u_{i-1}}, \quad \frac{\bar{u}_{i+1 / 2}-u_{i-1}}{u_{i}-u_{i-1}}, \quad \frac{\underline{u}_{i+1 / 2}-\underline{u}_{i-1 / 2}}{u_{i+1}-u_{i}}, \quad \frac{u_{i+1}-\underline{u}_{i-1 / 2}}{u_{i+1}-u_{i}}
$$

are always located between 0 and $\frac{3}{2}$.

The proofs being analogous, we will do the demonstration only for the first ratio, which will be denoted by $Q_{i}$ :

(a) If $\left(u_{i-1}, u_{i}, u_{i+1}\right)$ is not monotone, then (2.3) implies that $\bar{u}_{i+1 / 2}=u_{i}$ and $Q_{i} \in[0,1]$.

(b) If $\left(u_{i-1}, u_{i}, u_{i+1}\right)$ is monotone, we see with (2.3) that $Q_{i} \geq 0$, but in order to prove $Q_{i} \leq \frac{3}{2}$, we have to consider separately the cases $u_{i-1}<u_{i} \leq u_{i+1}$ and $u_{i+1} \leq u_{i}<u_{i-1}$. We detail only the first one:

$$
\begin{aligned}
Q_{i} & =\frac{\min \left(\hat{u}_{i+1 / 2}, u_{i+1}\right)-\bar{u}_{i-1 / 2}}{u_{i}-u_{i-1}} \quad(\text { see }(2.3)) \\
& \leq \frac{\hat{u}_{i+1 / 2}-\bar{u}_{i-1 / 2}}{u_{i}-u_{i-1}}=\frac{1}{2}+\frac{u_{i}-\bar{u}_{i-1 / 2}}{u_{i}-u_{i-1}} \quad(\text { see }(2.4)) \\
& \leq \frac{3}{2} \quad\left(\text { because } \bar{u}_{i-1 / 2} \in\left[u_{i-1}, u_{i}\right]\right) .
\end{aligned}
$$

4.2. Incremental decomposition. According to the notation (3.4), we have to find the incremental decomposition corresponding to the expression denoted $[D]=\lambda\left(f_{i+1 / 2}^{\mathrm{DA}}-f_{i-1 / 2}^{\mathrm{DA}}\right)$.

Let us start with the proof of Theorem 3.1: there are three possible definitions of the flux $f^{\mathrm{DA}}$ according to the value of $f^{\mathrm{G}}\left(u_{i+1 / 2}, u_{i}\right)$ (see (2.4)). For the sake of simplicity, we will describe only two cases among the nine total cases. The proof in the other cases is straightforward.

(a) First case: $f^{\mathrm{G}}\left(u_{i}, u_{i-1}\right)=f\left(u_{i-1}\right)$ and $f^{\mathrm{G}}\left(u_{i+1}, u_{i}\right)=f\left(u_{i}\right)$. Then

$$
\begin{aligned}
{[D] } & =\lambda\left(f^{\mathrm{G}}\left(u_{i+1}, \bar{u}_{i+1 / 2}\right)-f^{\mathrm{G}}\left(u_{i}, \bar{u}_{i-1 / 2}\right)\right) \\
& =-\left(u_{i+1}-u_{i}\right)[D 11]+\left(\bar{u}_{i+1 / 2}-\bar{u}_{i-1 / 2}\right)[D 12]
\end{aligned}
$$


with

$$
\begin{aligned}
& {[D 11]=\lambda \frac{f^{\mathrm{G}}\left(u_{i}, \bar{u}_{i+1 / 2}\right)-f^{\mathrm{G}}\left(u_{i+1}, \bar{u}_{i+1 / 2}\right)}{u_{i+1}-u_{i}},} \\
& {[D 12]=\lambda \frac{f^{\mathrm{G}}\left(u_{i}, \bar{u}_{i+1 / 2}\right)-f^{\mathrm{G}}\left(u_{i}, \bar{u}_{i-1 / 2}\right)}{\bar{u}_{i+1 / 2}-\bar{u}_{i-1 / 2}} .}
\end{aligned}
$$

Since the Godunov flux is increasing, we have $[D 11] \geq 0$ and $[D 12] \geq 0$; there exists $\theta \in I(u, v)$ such that $f^{\mathrm{G}}(u, v)=f(\theta)$; this allows us to bound [D11] and $[D 12]$ by

$$
\begin{cases}{[D 11] \leq \lambda\left|f^{\prime}\left(\theta_{1}\right)\right|} & \text { with } \theta_{1} \in I\left(u_{i}, u_{i+1}\right), \\ {[D 12] \leq \lambda\left|f^{\prime}\left(\theta_{2}\right)\right|} & \text { with } \theta_{2} \in I\left(\bar{u}_{i-1 / 2}, \bar{u}_{i+1 / 2}\right) \subset I\left(u_{i-1}, u_{i}, u_{i+1}\right),\end{cases}
$$

because $u_{i} \in I\left(\bar{u}_{i-1 / 2}, \bar{u}_{i+1 / 2}\right)$. Then, as in (3.4), we define the coefficients $C_{i+1 / 2}$ and $D_{i-1 / 2}$ by

$$
C_{i+1 / 2}=[D 11], \quad D_{i-1 / 2}=\frac{\bar{u}_{i+1 / 2}-\bar{u}_{i-1 / 2}}{u_{i}-u_{i-1}}[D 12] .
$$

Lemma 4.1 gives the following bounds:

$$
\left\{\begin{array}{l}
0 \leq C_{i+1 / 2} \leq \lambda \sup \left\{\left|f^{\prime}(u)\right|, u \in I\left(u_{i}, u_{i+1}\right)\right\} \\
0 \leq D_{i-1 / 2} \leq \frac{3}{2} \lambda \sup \left\{\left|f^{\prime}(u)\right|, u \in I\left(u_{i-1}, u_{i}, u_{i+1}\right)\right\}
\end{array}\right.
$$

(b) Second case: $f^{\mathrm{G}}\left(u_{i}, u_{i-1}\right)=f\left(u_{i}\right)$ and $f^{\mathrm{G}}\left(u_{i+1}, u_{i}\right)=f\left(\xi_{i+1 / 2}\right) \notin$ $\left\{f\left(u_{i}\right), f\left(u_{i+1}\right)\right\}$. Then

$$
[D]=\lambda\left(f\left(\xi_{i+1 / 2}\right)-f^{G}\left(\underline{u}_{i-1 / 2}, u_{i-1}\right)\right) .
$$

Here, the assumption of convexity on $f$ is essential to obtain $u_{i}<\xi_{i+1 / 2}<u_{i+1}$ from the property $f^{G}\left(u_{i+1}, u_{i}\right) \notin\left\{f\left(u_{i}\right), f\left(u_{i+1}\right)\right\}$. In order to decompose $[D]$, we have to consider the two cases $u_{i} \leq u_{i-1}$ and $u_{i-1}<u_{i}$. By using the same kind of arguments as above, we find when $u_{i} \leq u_{i-1}$ :

$$
0 \leq C_{i+1 / 2} \leq \lambda \sup \left\{\left|f^{\prime}(u)\right|, u \in I\left(u_{i}, u_{i+1}\right)\right\}, \quad D_{i-1 / 2}=0
$$

and when $u_{i-1}<u_{i}$ :

$$
0 \leq C_{i+1 / 2} \leq \frac{3}{2} \lambda \sup \left\{\left|f^{\prime}(u)\right|, u \in I\left(u_{i-1}, u_{i}, u_{i+1}\right)\right\}, \quad D_{i-1 / 2}=0
$$

Finally, by taking into account the nine possible majorations of $C_{i+1 / 2}$ and $D_{i+1 / 2}$, we obtain

$$
\begin{cases}0 \leq C_{i+1 / 2} \leq \frac{3}{2} \lambda \sup \left\{\left|f^{\prime}(u)\right|,\right. & \left.u \in I\left(u_{i-1}, u_{i}, u_{i+1}\right)\right\} \\ 0 \leq D_{i-1 / 2} \leq \frac{3}{2} \lambda \sup \left\{\left|f^{\prime}(u)\right|,\right. & \left.u \in I\left(u_{i-1}, u_{i}, u_{i+1}\right)\right\}\end{cases}
$$

which implies

$$
C_{i+1 / 2}+D_{i \pm 1 / 2} \leq 3 \lambda \sup \left|f^{\prime}\right| .
$$


Moreover, since $f$ is convex, $\left|f^{\prime}\right|$ is piecewise monotone and reaches its upper bound on the interval $I\left(u_{i-1}, u_{i}, u_{i+1}\right)$ at the boundaries (the values $\left.\left(u_{i}\right)_{i \in \mathbb{Z}}\right)$. Hence, we have the inequality

$$
C_{i+1 / 2}+D_{i \pm 1 / 2} \leq 3 \lambda \sup _{i \in \mathbb{Z}}\left|f^{\prime}\left(u_{i}\right)\right| .
$$

Under CFL condition $\lambda \sup _{i \in \mathbb{Z}}\left|f^{\prime}\left(u_{i}\right)\right| \leq \frac{1}{3}$, it follows from the above results that the two-point upstream weighting scheme is TVD and $L^{\infty}$-stable.

According to the property (4.3), the incremental constants are only depending on the $L^{\infty}$-stability majoration of the scheme, which proves, with (3.4), that the flux $f^{\mathrm{DA}}$ is "locally Lipschitz ${ }^{*}$."

The proof of Theorem 3.2 is similar to the proof above, but since $f$ is assumed monotone, the definition of the numerical flux $f^{\mathrm{DA}}$ becomes simpler: $f_{i+1 / 2}^{\mathrm{DA}}$ is equal to $f\left(\bar{u}_{i+1 / 2}\right)$ if $f$ is increasing, or $f\left(\underline{u}_{i+1 / 2}\right)$ if $f$ is decreasing.

If we assume, for instance, that $f$ is increasing, $\lambda\left(f\left(\bar{u}_{i+1 / 2}\right)-f\left(\bar{u}_{i-1 / 2}\right)\right)$ is the only possible value of $[D]$, and similarly to the computations done in $(4.2 \mathrm{a})$, we find in (4.1) and in (4.3) that $C_{i+1 / 2}=[D 11]=0$, which finally leads to

$$
C_{i+1 / 2}+D_{i \pm 1 / 2} \leq \frac{3}{2} \lambda \sup \left|f^{\prime}\right|
$$

Thus, we have a CFL condition which is two times less restrictive than the previous one.

\section{CONVERgence to the ENTROPY SOlUtion}

In order to prove that the weak solution $u$ obtained in Theorems 3.1 and 3.2 is the entropy solution, we must verify the Lax entropy condition (1.3) for all entropy convex $U(u)$. It is well known that this is satisfied whenever the scheme is consistent with the entropy condition for all convex entropy functions $U$. We prove the two following theorems.

Theorem 5.1. If $u_{0} \in L^{1}(\mathbb{R}) \cap L^{\infty}(\mathbb{R}) \cap B V(\mathbb{R})$, then for a convex function $f$, under the CFL condition $\lambda \sup \left\{\left|f^{\prime}\left(u_{i}\right)\right|, i \in \mathbb{Z}\right\} \leq \frac{1}{3}$, by using a slope limiter depending on the space step, the fully discrete two-point upstream weighting scheme converges to the entropy solution of the problem (1.1).

Theorem 5.2. If $u_{0} \in L^{1}(\mathbb{R}) \cap L^{\infty}(\mathbb{R}) \cap B V(\mathbb{R})$, then for a monotone function $f$, under the CFL condition $\lambda \sup \left|f^{\prime}\right| \leq \frac{2}{3}$, by using a slope limiter depending on the space step, the fully discrete two-point upstream weighting scheme converges to the entropy solution of the problem (1.1).

\section{Proof of Theorems 5.1 AND 5.2}

Convergence to the entropy solution is easily obtained by enforcing the scheme to be sufficiently close to an entropic scheme (that is to say, a scheme that is consistent with the entropy solution), as in [5], by means of a slope limiter. 
Applying convergence results of [12], we have convergence to the entropy solution as soon as the scheme can be written in the form

$$
\left\{\begin{array}{l}
u_{i}^{n+1}=\bar{u}_{i}^{n+1}-\left(a_{i+1 / 2}^{n}-a_{i-1 / 2}^{n}\right), \\
\bar{u}_{i}^{n+1}=u_{i}^{n}-\lambda\left(g_{i+1 / 2}^{n}-g_{i-1 / 2}^{n}\right),
\end{array}\right.
$$

where $a_{i+1 / 2}^{n}$ is a numerical flux going to zero when the space step $\Delta x$ tends to zero (for instance, $\left|a_{i+1 / 2}^{n}\right|<C \Delta x^{\alpha}$, with $\alpha \in(0,1)$, and $C$ a constant independent of $\Delta x)$, and $g_{i+1 / 2}^{n}$ is a monotone flux.

In the case of the two-point upstream weighting scheme, we choose $a_{i+1 / 2}^{n}=$ $\lambda\left(f_{i+1 / 2}^{\mathrm{DA}, n}-f^{\mathrm{G}}\left(u_{i+1}^{n}, u_{i}^{n}\right)\right)$ and $g_{i+1 / 2}^{n}=f_{i+1 / 2}^{\mathrm{G}}$, which is obviously monotone. The only condition to be verified is then $\left|a_{i+1 / 2}^{n}\right|<C \Delta x^{\alpha}$, which is satisfied under the following slope limiter:

$$
\begin{cases}\left|\bar{u}_{i+1 / 2}-u_{i}\right| \leq C(\Delta x)^{\alpha} & \text { when } f^{\mathrm{G}}\left(u_{i+1}, u_{i}\right)=f\left(u_{i}\right), \\ \left|\underline{u}_{i+1 / 2}-u_{i+1}\right| \leq C(\Delta x)^{\alpha} & \text { when } f^{\mathrm{G}}\left(u_{i+1}, u_{i}\right)=f\left(u_{i+1}\right),\end{cases}
$$

for both Theorems 5.1 and 5.2.

Remark. The condition $\left|a_{i+1 / 2}\right|<C \Delta x^{\alpha}$ is a slope limiter depending on the space step $\Delta x$, which could be rather restrictive when $\Delta x$ tends to zero (except if we are in a smooth region of the solution, because then $\left.\left|a_{i+1 / 2}\right|=O(\Delta x)\right)$. However, in numerical computations, if $\Delta x$ is fixed, this slope limiter will never be active because there exists a sufficiently small $\alpha$ such that the inequality is always verified.

\section{Part II. Convergence of the Semidiscrete \\ Two-Point Upstream Weighting Scheme TO THE ENTROPY SOLUTION}

\section{CONVERGENCE TO A WEAK SOlution}

The semidiscrete scheme (1.5) is an infinite-dimensional differential system (because $i \in \mathbb{Z}$ ). We have to prove the existence of a solution, and then, to prove that this solution is a weak solution.

The most important problem about proving existence of a solution of (1.5) is to have a globally Lipschitz numerical flux and to have $u$ belonging to a suitable Banach space in order to apply the Cauchy theorem. First we prove (Lemma 8.1) that the flux $f^{\mathrm{DA}}$ is globally Lipschitz under monotonicity and regularity assumptions. Next, we exhibit a sufficient condition on the initial data $u_{0}$ to reduce the general semidiscrete scheme $(1.5)$ to the resolution of a finite-dimensional differential system,

$$
u_{i}(0)= \begin{cases}C_{g} & \text { if } i \leq I_{g} \\ C_{d} & \text { if } i \geq I_{d}\end{cases}
$$

where $C_{g}$ and $C_{d}$ are constants and $I_{g}<I_{d}$. Then, assuming the numerical flux associated with the scheme (1.5) is globally Lipschitz, we apply the Cauchy 
theorem to prove (Lemma 8.2) the existence of a solution $\left(u_{i}\right)_{i \in \mathbb{Z}}$ verifying the same kind of property as $u_{0}$ in (7.1).

Remark. The assumption (7.1) with $C_{g}=C_{d}=0$ means that $u_{0}$ has compact support, which is a very classical hypothesis that allows one to solve Riemann's problems.

Finally, we give sufficient conditions for the solution of (1.5) to be a weak solution, that is to say, for the scheme to be TVD (Lemma 8.3) and $L^{\infty}$-stable (Lemma 8.4).

Applying these lemmas to the two-point upstream weighting scheme, we then prove:

Theorem 7.1. If $u_{0} \in L^{1}(\mathbb{R}) \cap B V(\mathbb{R})$, and assuming.

- $u_{0}$ satisfies (7.1),

- the function $f$ is monotone and $\left|f^{\prime}\right|$ is bounded,

then the semidiscrete two-point upstream weighting scheme converges to a weak solution of the problem (1.1).

\section{Proof of Theorem 7.1}

After proving the preliminary lemmas, we will conclude the proof in $\S 8.3$.

\subsection{Conditions on $f$ for the flux $f^{\mathrm{DA}}$ to be globally Lipschitz.}

Lemma 8.1. If the function $f$ is monotone, and if $\left|f^{\prime}\right|$ is bounded, then the numerical flux $f^{\mathrm{DA}}$ is globally Lipschitz.

The flux $f^{\mathrm{DA}}$ is (globally) Lipschitz if there exists a constant $C>0$ such that

$$
\begin{gathered}
\forall U\left(u_{i-1}, u_{i}, u_{i+1}, u_{i+2}\right) \in \mathbb{R}^{4}, \forall V\left(v_{i-1}, v_{i}, v_{i+1}, v_{i+2}\right) \in \mathbb{R}^{4}, \\
\left|f^{\mathrm{DA}}(U)-f^{\mathrm{DA}}(V)\right| \leq C\|U-V\|_{\mathbb{R}^{4}}
\end{gathered}
$$

Proof of Lemma 8.1. In [10], it is proved that $f^{\mathrm{DA}}$ is not globally Lipschitz if $f^{G}\left(u_{i+1}, u_{i}\right) \notin\left\{f\left(u_{i}\right), f\left(u_{i+1}\right)\right\}$. By removing this possibility, we are led to assume that $f$ is monotone. To simplify the proof, we assume in the following that the function $f$ is increasing. We are going to prove that

$$
\begin{aligned}
\left|f^{\mathrm{DA}}(U)-f^{\mathrm{DA}}(V)\right| & =\left|f\left(\bar{u}_{i+1 / 2}\right)-f\left(\bar{v}_{i+1 / 2}\right)\right| \\
& \leq\left|\bar{u}_{i+1 / 2}-\bar{v}_{i+1 / 2}\right| \cdot \sup \left\{\left|f^{\prime}(u)\right|, u \in I\left(\bar{u}_{i+1 / 2}, \bar{v}_{i+1 / 2}\right)\right\} \\
& \leq 2\|U-V\|_{\mathbb{R}^{4}} \cdot \sup \left\{\left|f^{\prime}(u)\right|, u \in I\left(u_{i}, u_{i+1}, v_{i}, v_{i+1}\right)\right\},
\end{aligned}
$$

which will prove Lemma 8.1 thanks to the assumption on $\left|f^{\prime}\right|$.

In order to prove the above inequalities, we need only verify that $[\bar{d}]=$ $\left|\bar{u}_{i+1 / 2}-\bar{v}_{i+1 / 2}\right| \leq 2\|U-V\|_{\mathbb{R}^{4}}$. 
There are three possible definitions of $\bar{u}_{i+1 / 2}$ and $\bar{v}_{i+1 / 2}$, and nine values of the difference. We detail the case $u_{i-1} \leq u_{i} \leq u_{i+1}$ and $v_{i-1} \leq v_{i} \leq v_{i+1}$ (the other cases of (2.3) are straightforward). Then

$$
[\bar{d}]=\left|\min \left\{\hat{u}_{i+1 / 2}, u_{i+1}\right\}-\min \left\{\hat{v}_{i+1 / 2}, v_{i+1}\right\}\right| .
$$

If $\hat{u}_{i+1 / 2} \leq u_{i+1}$ and $\hat{v}_{i+1 / 2} \leq v_{i+1}$, then

$$
\begin{aligned}
{[\bar{d}] } & =\left|\hat{u}_{i+1 / 2}-\hat{v}_{i+1 / 2}\right|=\left|\frac{3}{2} u_{i}-\frac{1}{2} u_{i-1}-\frac{3}{2} v_{i}+\frac{1}{2} v_{i-1}\right| \\
& \leq \frac{3}{2}\left|u_{i}-v_{i}\right|+\frac{1}{2}\left|u_{i-1}-v_{i-1}\right| \leq 2\|U-V\| .
\end{aligned}
$$

If $\hat{u}_{i+1 / 2} \leq u_{i+1}$ and $v_{i+1} \leq \hat{v}_{i+1 / 2}$, then

$$
\begin{aligned}
{[\bar{d}] } & =\left|\hat{u}_{i+1 / 2}-v_{i+1}\right|=\hat{u}_{i+1 / 2}-v_{i+1} \quad\left(\text { if } v_{i+1} \leq \hat{u}_{i+1 / 2}\right) \\
& \leq u_{i+1}-v_{i+1} \leq\|U-V\| .
\end{aligned}
$$

If $u_{i+1} \leq \hat{u}_{i+1 / 2}$ and $\hat{v}_{i+1 / 2} \leq v_{i+1}$, then

$$
\begin{aligned}
{[\bar{d}] } & =\left|u_{i+1}-\hat{v}_{i+1 / 2}\right|=u_{i+1}-\hat{v}_{i+1 / 2} \quad\left(\text { if } \hat{v}_{i+1 / 2} \leq u_{i+1}\right) \\
& \leq \hat{u}_{i+1 / 2}-\hat{v}_{i+1 / 2} \leq 2\|U-V\| \quad \text { (see above). }
\end{aligned}
$$

If $u_{i+1} \leq \hat{u}_{i+1 / 2}$ and $v_{i+1} \leq \hat{v}_{i+1 / 2}$, then

$$
[\bar{d}]=\left|u_{i+1}-v_{i+1}\right| \leq\|U-V\| .
$$

\subsection{Existence of a solution of (1.5).}

Lemma 8.2. If $u_{0}$ satisfies property (7.1) and if the numerical flux $g$ is globally Lipschitz, then the differential system associated with the scheme (1.5) has a solution satisfying

$$
u_{i}(t)= \begin{cases}C_{g} & \text { if } i \leq I_{g}-k, \\ C_{d} & \text { if } i \geq I_{d}+k,\end{cases}
$$

where $2 k$ is the number of arguments in the numerical flux $g$.

Proof. Let $(S)$ denote the subsystem of (1.5) obtained by taking into account the equations from $i=I_{g}-k+1$ to $i=I_{d}+k-1$. Thanks to the Cauchy theorem, if the numerical flux $g$ is Lipschitz, then $(S)$ has a unique solution associated with the initial data $u_{0}$. Extending this solution by $u_{i}(t)=C_{g}$ if $i \leq I_{g}-k$ and $u_{i}(t)=C_{d}$ if $i \geq I_{d}+k$, under the assumption (7.1) on $u_{0}$, the new sequence $\left(u_{i}\right)_{i \in \mathbb{Z}}$ is a solution of (1.5) (because the flux $g$ is consistent) that satisfies $(8.1)$.

Remark. We prove in [10] the existence and uniqueness of the solution of the infinite-dimensional system without using the assumption (7.1), but we are led to assume that the scheme is TVD. 
8.3. Sufficient condition for the scheme (1.5) to be TVD and $L^{\infty}$-stable. As we did in the fully discrete case, we use the following incremental form of the scheme (1.5):

$$
\frac{1}{\Delta x}\left(g_{i+1 / 2}-g_{i-1 / 2}\right)=-C_{i+1 / 2}^{s}\left(u_{i+1}-u_{i}\right)+D_{i-1 / 2}^{s}\left(u_{i}-u_{i-1}\right),
$$

where $C_{i+1 / 2}^{s}$ and $D_{i+1 / 2}^{s}$ depend on $\left(u_{i-k+1}, \ldots, u_{i+k}\right)$. Hence, with (8.2), the scheme (1.5) becomes

$$
\frac{d u_{i}}{d t}=C_{i+1 / 2}^{s}\left(u_{i+1}-u_{i}\right)-D_{i-1 / 2}^{s}\left(u_{i}-u_{i-1}\right) .
$$

Because of Lemma 8.2, we know that the solution of (1.5) satisfies (8.1), which reduces the total variation $\frac{d}{d t} \sum_{i}\left|u_{i+1}(t)-u_{i}(t)\right|$ to a finite series. Then the following holds.

Lemma 8.3. If $u_{0}$ satisfies property (7.1), and the scheme (1.5) can be written in incremental form (8.3) with coefficients $C_{i+1 / 2}^{s} \geq 0$ and $D_{i-1 / 2}^{s} \geq 0$, then the scheme is TVD.

This is proved by applying a result of S. Osher and S. Chakravarthy [7] valid in finite dimensions: if a scheme (1.5) (with $i$ describing a finite domain) can be written in incremental form (8.3) with coefficients $C_{i+1 / 2}^{s} \geq 0$ and $D_{i-1 / 2}^{s} \geq 0$, then the scheme is TVD.

Taking into account Lemma 8.3, it is easy to prove:

Lemma 8.4. Under the assumptions of Lemma 8.3, if $u_{0} \in B V(\mathbb{R})$, then the scheme (1.5) is $L^{\infty}$-stable.

Indeed, the scheme is TVD and property (8.1) implies

$$
u_{i}=\sum_{j \leq i}\left(u_{j}-u_{j-1}\right)+C_{g} \text {. }
$$

We prove easily that the two-point upstream weighting scheme can be written in incremental form with positive incremental coefficients by comparing the incremental forms in (3.4) and (8.2). As a matter of fact, the analogy between the couples $\left(C_{i+1 / 2}, D_{i-1 / 2}\right)$ and $\left(C_{i+1 / 2}^{s}, D_{i-1 / 2}^{s}\right)$ allows us to take into account all the results of $\S 4$ and to deduce that $C_{i+1 / 2}^{s} \geq 0$ and $D_{i+1 / 2}^{s} \geq 0$.

Assuming that $u_{0}$ satisfies $(7.1)$ and $u_{0} \in B V(\mathbb{R})$, we deduce from the lemmas above that the two-point upstream weighting scheme is TVD and $L^{\infty}$ stable. Because of Lemma 8.1 , the flux $f^{\mathrm{DA}}$ is Lipschitz under the assumptions of Theorem 7.1. Applying now the same result as in the fully discrete case, we deduce that the two-point upstream weighting scheme converges to a weak solution as soon as we assume $u_{0} \in L^{1}(\mathbb{R}) \cap B V(\mathbb{R})$.

\section{THEOREM OF CONVERGENCE TO THE ENTROPY SOLUTION}

Under the convergence to a weak solution hypothesis, if $f$ is convex and, moreover, the solution has bounded variation, the consistency of the scheme 
with the entropy condition (which implies the Lax entropy condition) associated with a strictly convex function $U$, implies the convergence to the unique entropy solution. We prove:

Theorem 9.1. If $u_{0} \in L^{1}(\mathbb{R}) \cap B V(\mathbb{R})$, if $u_{0}$ satisfies property (7.1) and, if the function $f$ is convex monotone and $\left|f^{\prime}\right|$ bounded, then, by using a slope limiter independent of the space step, the semidiscrete two-point upstream weighting scheme converges to the entropy solution of the problem (1.1).

\section{Proof of Theorem 9.1}

To prove that the scheme is consistent with the Lax entropy condition, we have to verify the following entropy inequality:

$$
\frac{d}{d t} U\left(u_{i}\right)+\frac{1}{\Delta x}\left(G_{i+1 / 2}-G_{i-1 / 2}\right) \leq 0,
$$

where $G$ is a continuous function, $G_{i+1 / 2}$ denotes $G\left(u_{i-k+1}, \ldots, u_{i+k}\right)$, and $G$ is such that $G(u, \ldots, u)=F(u)$.

To do this, we need only choose a suitable function $G_{i+1 / 2}$, for instance, as in [6],

$$
G_{i+1 / 2}(t)=F\left(u_{i+1}(t)\right)+U^{\prime}\left(u_{i}(t)\right) \cdot\left(g_{i+1 / 2}(t)-f\left(u_{i+1}(t)\right)\right),
$$

which gives

$$
\begin{aligned}
\frac{d}{d t} U & \left(u_{i}(t)\right)+\frac{1}{\Delta x}\left(G_{i+1 / 2}(t)-G_{i-1 / 2}(t)\right) \\
& =\frac{1}{\Delta x} \int_{u_{i}(t)}^{u_{i+1}(t)} U^{\prime \prime}(u) \cdot\left(g_{i+1 / 2}(t)-f(u)\right) d u .
\end{aligned}
$$

This reduces the problem of convergence to the entropy solution to the computation of an integral.

Remark. The entropy (10.1), with (10.2), allows us to obtain directly, as in [12], the convergence of the fully discrete implicit scheme associated with (1.4).

To verify the entropy inequality associated with the two-point upstream weighting scheme, we choose $U(u)=u^{2} / 2$. The relation of consistency with the entropy condition associated with $U(u)$ becomes (with $f$ increasing for instance):

$$
\int_{u_{i}}^{u_{i+1}}\left(f\left(\bar{u}_{i+1 / 2}\right)-f(u)\right) d u \leq 0 .
$$

Hence, the proof of Theorem 9.1 consists in finding a suitable correction by means of a slope limiter [10] to enforce $\bar{u}_{i+1 / 2}$ to satisfy the above inequality, for instance, as in [6]. But it is worth noticing that the correction used is entirely independent of the space step. 


\section{PART III. Numerical Results}

To illustrate the two-point upstream weighting scheme, we apply this scheme to the resolution of the Buckley-Leverett equation, which represents the displacement of an incompressible two-phase flow in a porous medium. The flux function $f(u)$ represents the so-called fractional flow, relating the mobility of displacing fluid to the total fluid mobility.

Numerical tests have been done, considering a high-mobility ratio displacement, which could give some numerical instabilities. We consider $f(u)=$ $20 u^{3} /\left(20 u^{3}+(1-u)^{3}\right)$, which describes the displacement of two immiscible fluids with cubic relative permeability and a viscosity ratio of 20 .

We compare below the solutions of the Riemann problem (with initial data $u_{0}(x)=1$ if $x<0$, and 0 if $x>0$ ) computed by the two-point upstream weighting explicit scheme and the Van Leer explicit scheme (other comparsions can be found in [10], especially between implicit versions of these schemes).

Figure 3 shows the solutions computed by the two schemes are very close; but we notice that the two-point upstream weighting scheme is slightly more effective than the Van Leer scheme in describing the shock front and less accurate in computing the rarefaction wave. In order to compare more easily the two schemes, we still give in Figures 4 and 5 the corresponding algebraic difference between the analytic solution and the computed solutions.

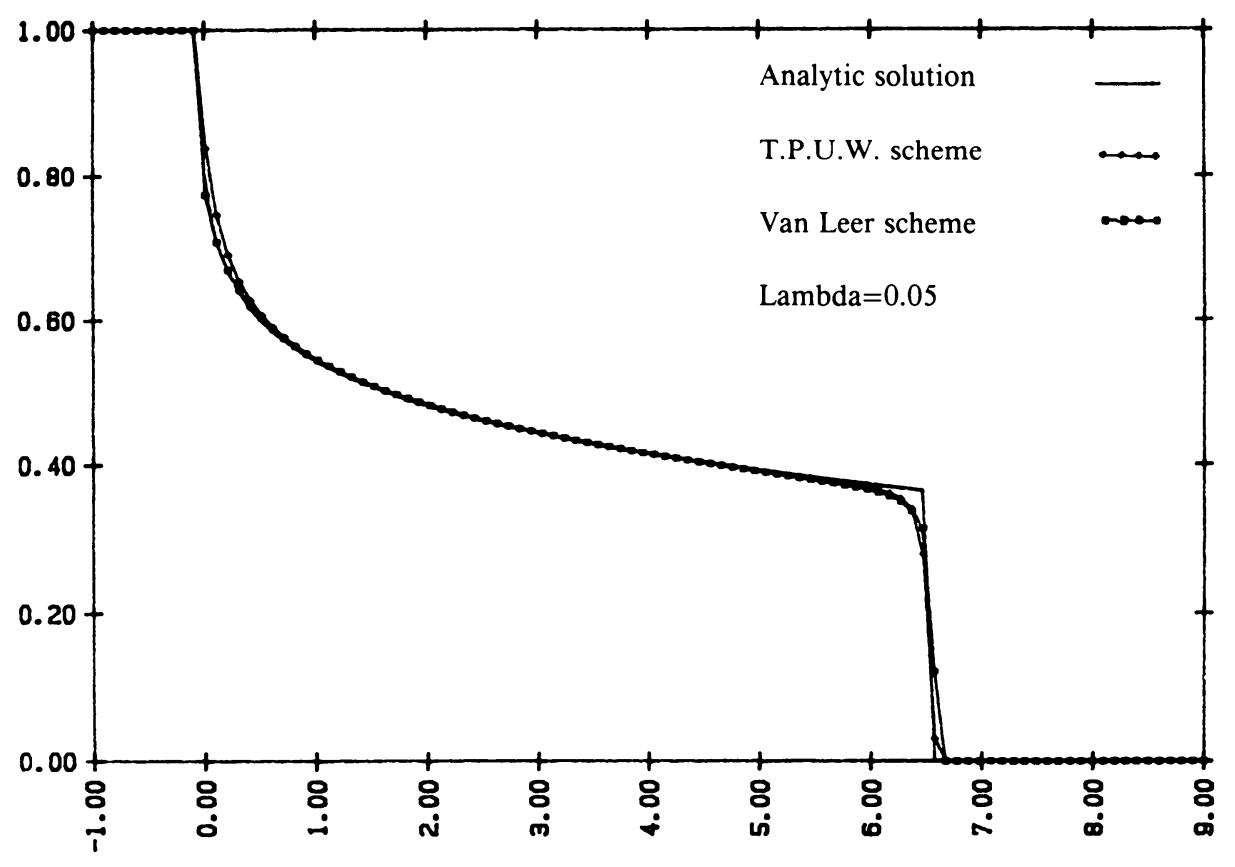

FIGURE 3 


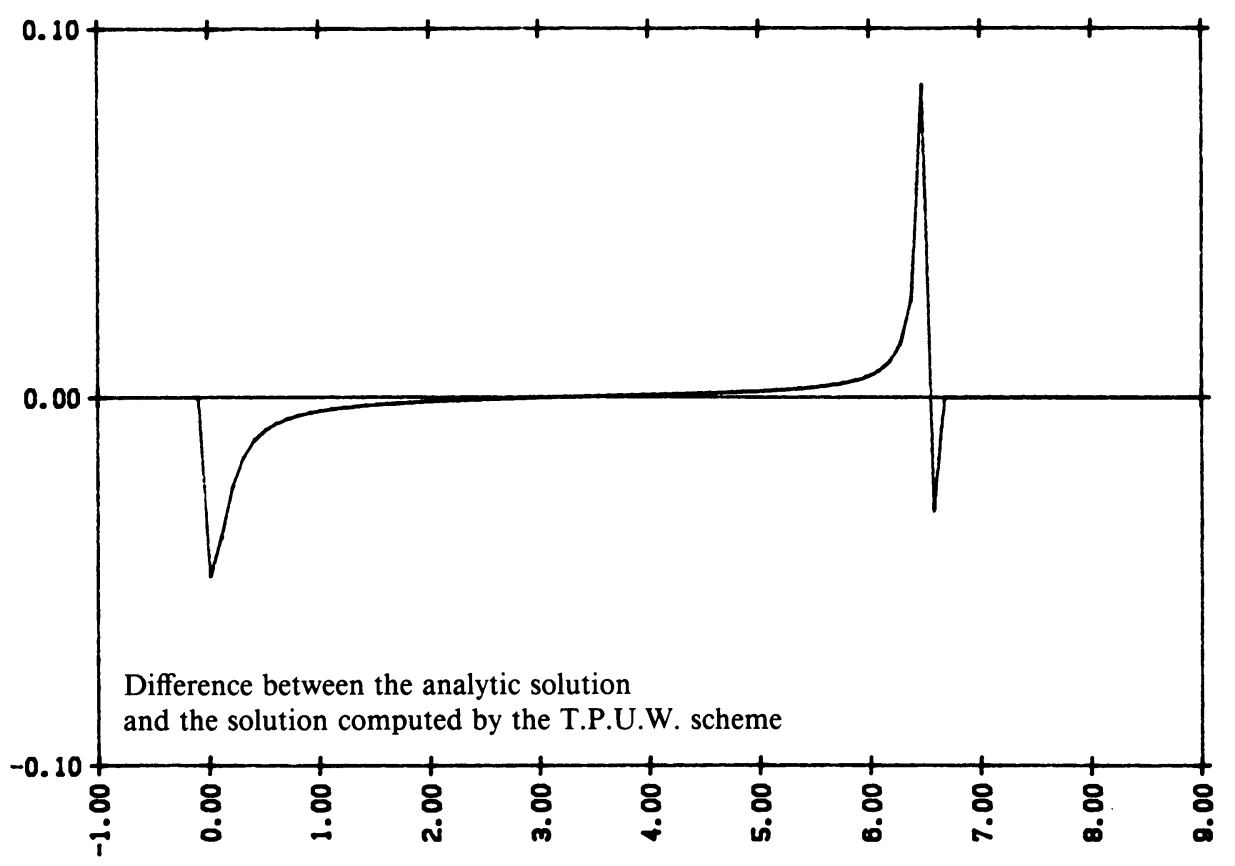

Figure 4

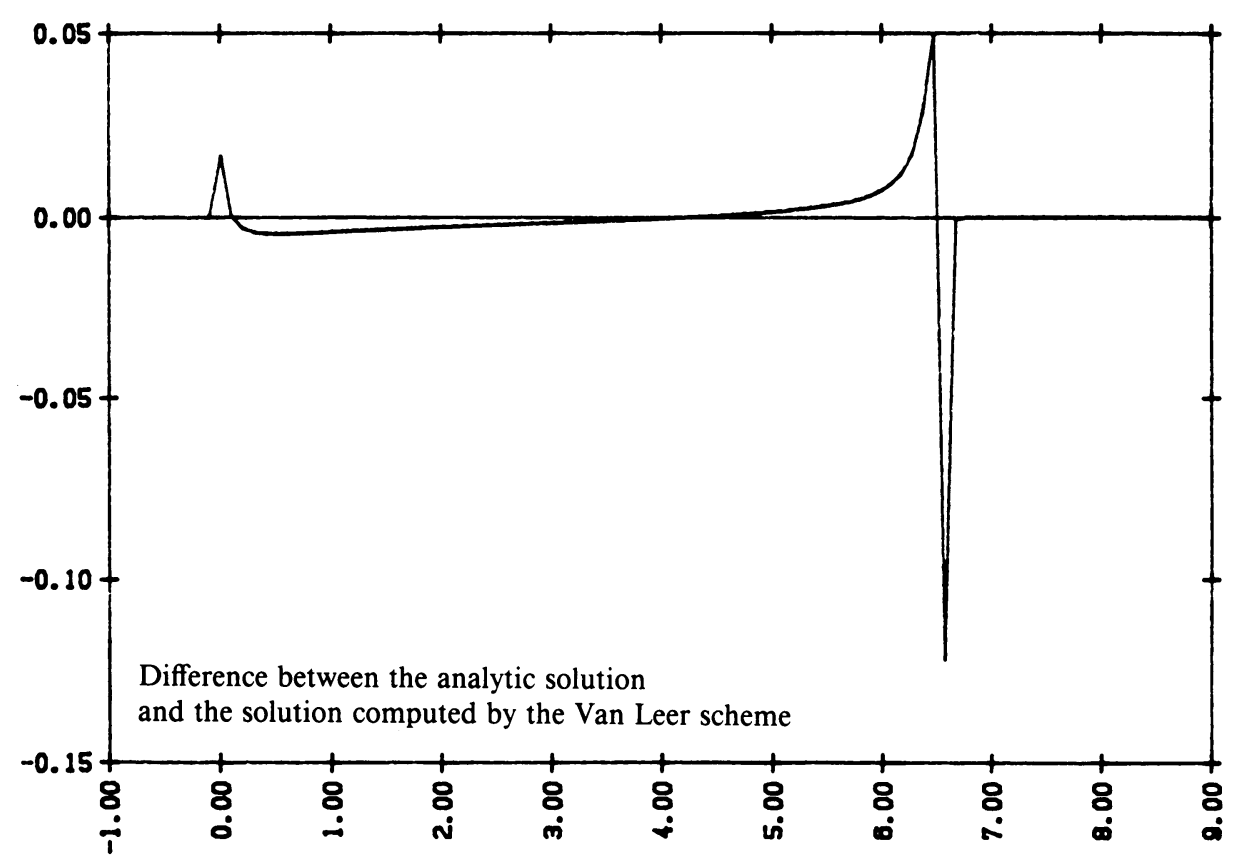

FIGURE 5 


\section{ACKNOWLEDGMENT}

This work forms part of the author's doctoral dissertation. He wants to express his deep gratitude to Professor Alain Bourgeat for encouraging guidance and for always being available for extensive discussions.

\section{BIBLIOGRAPHY}

1. A. Bourgeat, Rapport final du contrat GDF/Université de Saint-Etienne, Publication de l'Equipe d'Analyse Numérique, Lyon-Saint-Etienne, Juin 1987.

2. G. Chavent and B. Cockburn, Consistance et stablilité des schémas LRG pour les lois de conservation scalaires, Rapport de Recherche $\mathrm{N}^{\circ} 710$, INRIA, Centre de Rocquencourt, Juillet 1987.

3. R. J. Di Perna, Uniqueness of solutions to hyperbolic conservation laws, Indiana Univ. Math. J. 28 (1979), 137-188.

4. A. Harten, On a class of high resolution total-variation-stable finite-difference schemes, SIAM J. Numer. Anal. 21 (1984), 1-23.

5. A. Y. Le Roux, Convergence of an accurate scheme for first order quasi-linear equations, RAIRO Anal. Numér. 15 (1981), 151-170.

6. S. Osher, Convergence of generalized MUSCL schemes, SIAM J. Numer. Anal. 22 (1985), 947-961.

7. S. Osher and S. Chakravarthy, High resolution schemes and the entropy condition, SIAM J. Numer. Anal. 21 (1984), 955-984.

8. H. S. Price, R. S. Varga, and J. E. Warren, Application of oscillation matrices to diffusionconvection equations, J. Math Phys. 45 (1966), 301-311.

9. M. R. Todd, P. M. O’Dell, and G. J. Hirasaki, Methods for increased accuracy in numerical reservoir simulators, Trans. Soc. Petroleum Engrs. 253 (1972), 515-530 (Soc. Petroleum Engrs. J.).

10. M. C. Viallon, Etude des schémas Double-Amont et éléments finis discontinus $P^{0} P^{1}$ pour la résolution numérique des lois de conservation scalaires, Thèse, Université de Saint-Etienne, 1989.

11. J. P. Vila, High-order schemes and entropy condition for nonlinear hyperbolic systems of conservations laws, Math. Comp. 50 (1988), 53-73.

12. __ Sur la théorie et l'approximation numérique de problèmes hyperboliques non linéaires. Application aux équations de Saint Venant et à la modélisation des avalanches de neige dense. Thèse, Université Paris VI, 1986.

13. M. J. Wheatley, $A$ version of two-point upstream weighting for use in implicit numerical reservoir simulators, paper 7677 of the Fifth. Sympos. on Reservoir Simul., Soc. Petroleum Engrs. of AIME, Denver, 1979.

Equipe d'Analyse Numerique, Universite Saint-Etienne, 23 rue Paul Michelon, 42023 Saint-Etienne Cedex 2, France 